\title{
Electron Microscopy Analysis of Grain Boundary Corrosion in Ni-Cr Binary Alloys Exposed to High Temperature Hydrogenated Water
}

\author{
Matthew J. Olszta ${ }^{1}$, Daniel K. Schreiber ${ }^{1}$ and Stephen M. Bruemmer ${ }^{1}$ \\ 1. Energy and Environment Directorate, Pacific Northwest National Laboratory, PO Box, 999 Richland, \\ WA USA
}

Intergranular (IG) corrosion and stress corrosion cracking are important issues in current and advanced nuclear systems, and the understanding the controlling mechanisms are of great concern to the nuclear industry. In order to better elucidate fundamental aspects, this study focuses on exposure of coupons of varying $\mathrm{Cr}$ content in Ni-Cr binary alloys. Detailed evaluation of Cr concentration effects on protective film formation and localized corrosion provides underpinning processes controlling behavior of complex Ni-base alloys in light-water reactor service.

Coupons of Ni-5Cr, 10Cr, 20Cr and 30Cr binary alloys were polished to a colloidal silica finish to minimize surface damage and expose the bulk grain boundaries. The alloys were exposed to simulated pressurized water reactor primary water environments at $320-360^{\circ} \mathrm{C}$ for $500-3500 \mathrm{~h}$ [1]. After exposure, cross-sections of each specimen were characterized using low kV scanning electron microscopy (SEM) in backscatter mode as well as transmission and scanning transmission electron microscopy (TEM/STEM) using both electron energy loss and energy dispersive spectroscopies (EELS and EDS, respectively). Alloys with lower $\mathrm{Cr}$ concentrations (Ni-5Cr and $\mathrm{Ni}-10 \mathrm{Cr}$ ) exhibited IG corrosion to depths on the order of 10s of micrometers (with the deepest for each reaching $~ 30 \mu \mathrm{m}$ ), whereas when the Cr concentration reached 20 at\% the IG corrosion was observed on the order of a few micrometers. Typical examples of these corrosion structures are shown in Figure 1. At 30 at\% Cr, there was no IG corrosion, as a thin protective surface oxide was observed.

Representative TEM/STEM analyses at the leading exposure front for both the lowest and highest Cr content, Ni-based binary alloys is shown in Figures 2 and 3 illustrating $\mathrm{Cr}$ depletion and diffusion induced grain boundary migration (DIGM) [2]. At the leading IGA in the Ni-5Cr, the Cr depletion is on the order to 10s of nanometers wide with depletion as low as 0.5 at $\% \mathrm{Cr}$ (Figure 2). The final position of the grain boundary is observed in the annular darkfield (ADF) image adjacent to the oxidation front and the initial boundary position is indicated by the extent of the Cr depletion. ADF and energy filtered images of the Ni-30Cr illustrate that while DIGM also occurs on the order of hundreds of nanometers (with depletion down to 1-2 at\% Cr), there is no IG corrosion present (Figure 3). A thin film of chromia passivates the grain boundary and prevents localized corrosion. Analysis of the various morphologies and levels of depletion of $\mathrm{Cr}$ along grain boundaries assists in the understanding $\mathrm{Ni}-\mathrm{Cr}$ alloy performance in nuclear reactor components.

[1] D.K. Schreiber et al, Microscopy \& Microanalysis (2013), 19(3), 678-687.

[2] R.W. Balluffi and J.W. Cahn, Acta Metallurgica (1981), Vol. 29, 493-500.

The authors acknowledge funding from the Department of Energy, Office of Basic Energy Sciences under contract DE-AC06-76RLO 1830. 


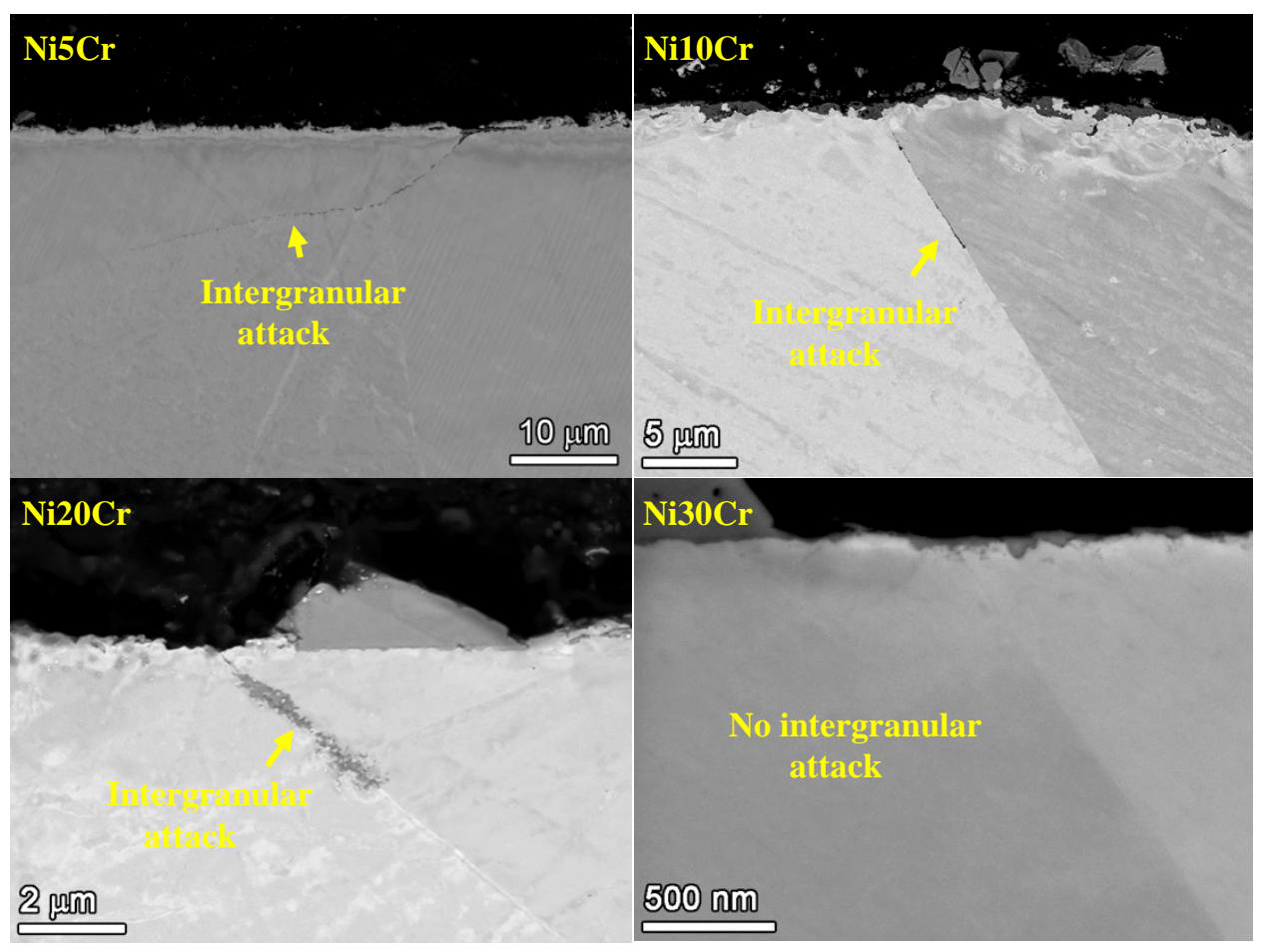

Figure 1. SEM backscatter images of Ni-5Cr, Ni-10Cr, Ni-20Cr and Ni-30Cr exposed at $600 \mathrm{~h}$ in hydrogenated water at $360^{\circ} \mathrm{C}$ illustrating the exposure depths (or lack thereof in Ni-30Cr).

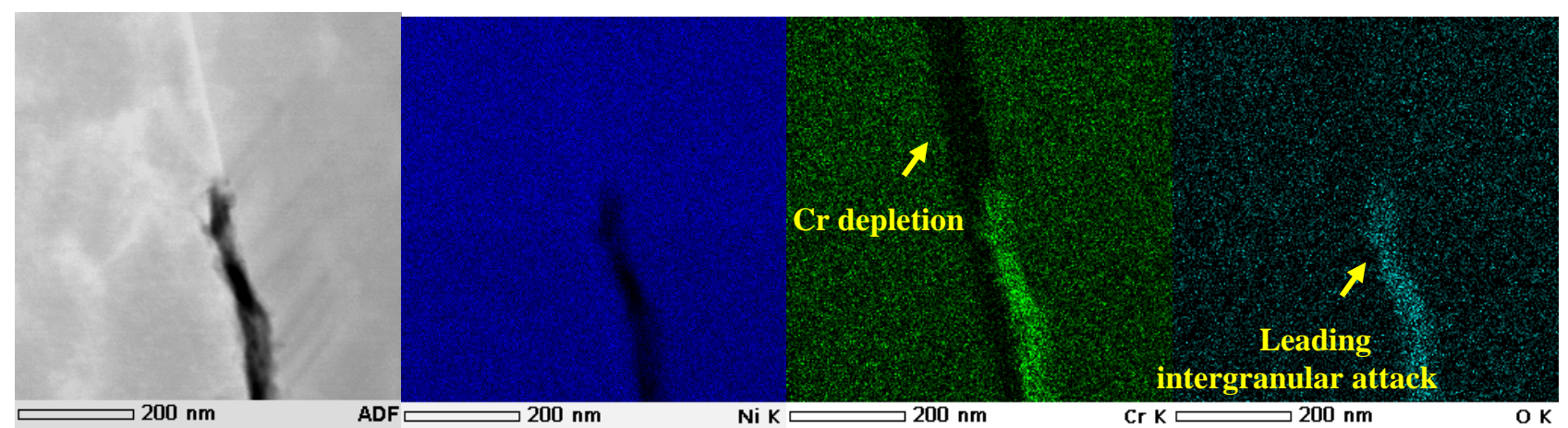

Figure 2. STEM EDS elemental maps of exposed Ni-5Cr showing the extent of Cr grain boundary depletion ahead (down to 0.5 at\%) of the leading intergranular attack front.
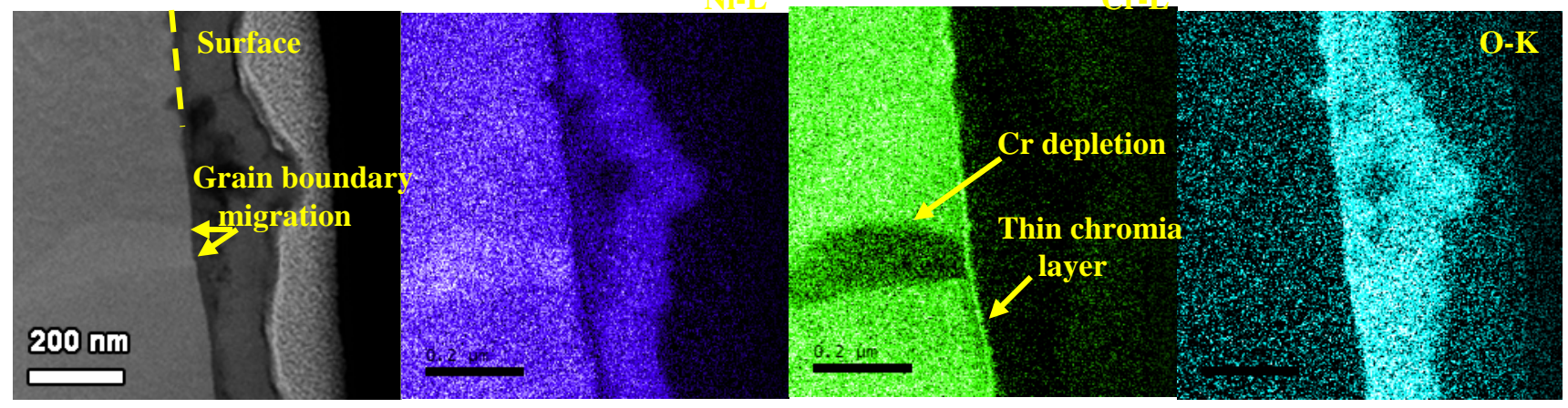

Figure 3. Annular DF and GIF elemental maps of exposed Ni-30Cr showing the extent of Cr grain boundary depletion near the surface as well as protective chromia formation above the grain boundary. 